\title{
Determinação por Espectroscopia nas Regiões MIR/NIR do Teor de NCO em Adesivos Poliuretânicos
}

\author{
Sandra H. S. Siqueira \\ Rohm and Haas Química Ltda e Instituto Tecnológico da Aeronáutica, ITA
}

Rita C.L. Dutra, Milton F. Diniz

Divisão de Química, Instituto de Aeronáutica e Espaço, CTA

\begin{abstract}
Resumo: Adesivos poliuretânicos (AD-PU), contendo teor conhecido de isocianato (NCO), foram preparados, nos laboratórios da Rohm and Haas Química Ltda, e amostras foram usadas para a elaboração de uma curva analítica, visando à determinação do teor de NCO por espectroscopia no infravermelho médio (MIR) e próximo (NIR). Os valores de \% NCO, obtidos pelo método analítico por via úmida para amostras retiradas durante o processo de fabricação, foram usados como referência. A banda FT-IR escolhida na região MIR foi: $2270 \mathrm{~cm}^{-1}$ (banda analítica). Na região NIR, foi usada uma banda relativa, $\mathrm{A}_{4063} / \mathrm{A}_{4348}$. Em ambos os métodos, MIR e NIR, a intensidade das bandas escolhidas e o teor de NCO, obtidos a partir da análise via úmida, foram utilizados para construção de curvas que apresentaram boas correlações lineares (MIR - R $=0,995$ e NIR - R = 0,961), que possibilitarão a determinação dos teores de NCO em misturas de composição semelhante.
\end{abstract}

Palavras-chave: Adesivos poliuretânicos, teor de NCO, infravermelho com transformada de Fourier (FT-IR), MIR, NIR.

\section{Determination of NCO Contents in Polyurethane Adhesives Using MIR/NIR Spectroscopies}

Abstract: Polyurethane adhesives reference samples (AD-PU) with known contents of isocyanate (NCO) have been prepared in Rohm and Haas to elaborate analytical curves for determination of NCO content, using MIR and NIR analysis. Wet chemical values were used to determine the content of NCO in the AD-PU samples obtained during the process. The MIR analytical band used was $2270 \mathrm{~cm}^{-1}$ and the NIR relative band was $\mathrm{A}_{4063} / \mathrm{A}_{4348}$. Data of absorbance intensity and NCO contents obtained from wet chemical analysis were used to elaborate the analytical curves (MIR $-\mathrm{R}=0.995$ and $\mathrm{NIR}-\mathrm{R}=0.961$ ) which should allow for the determination of NCO content in adhesive samples of similar composition.

Keywords: Polyurethanes adhesives, NCO content, FT-IR, MIR and NIR.

\section{Introdução}

Como é conhecido, o poliuretano (PU) foi descoberto por Otto Bayer e colaboradores em I. G. Farbenindustrie, Alemanha, por volta de 1930. O primeiro produto foi obtido pela reação de diisocianato alifático com diamina alifática ou diol e comercializados com os nomes de Irgamid U, para aplicações em plásticos e Perlon U para aplicações em fibras sintéticas ${ }^{[1]}$.

Logo após a descoberta do poliuretano, Otto Bayer descobriu que o isocianato poderia ser utilizado para unir borracha em metal, no qual por mais uma vez liderou o desenvolvimento do primeiro adesivo uretânico, baseado em poliéster diol; este adesivo foi comercializado com o nome de Polystal ${ }^{[1]}$. Os adesivos podem ser definidos como quaisquer substâncias capazes de unir dois materiais (papel, vidro, plástico, metal, cerâmica ou qualquer combinação destes materiais $)^{[2]}$. Atualmente, existem vários tipos de adesivos para diferentes setores industriais. Neste trabalho será focalizado os adesivos à base de PU, utilizados em aplicações de laminados impressos e não impressos como PETP, PA, PP, PE (incluindo tipos EVA) .

Os adesivos de PU polimerizam para formar ligações resistentes, sem a necessidade de altas temperaturas. As elevadas forças de ligação interfacial, obtidas, são derivadas, não somente das forças físicas resultantes do contato íntimo, mas também da habilidade do adesivo de PU, de formar ligações hidrogênio, ou ligações covalentes, com diferentes substratos, como na reação com grupos aminas ou amidas. Tais ligações têm excelente durabilidade, especialmente quando os adesivos de PU são feitos com polióis hidrofóbicos como os polióis poliéter e polibutadieno líquido. A principal razão da alta resistência à umidade da ligação uretânica com superfícies metálicas é atribuída à habilidade dos isocianatos de reagirem com uma camada unimolecular de água, presente na superfície metálico, como alumínio, anteriormente tratado superficialmente por processos padrões $^{[3]}$.

Pela utilização de diferentes matérias primas, as propriedades dos adesivos de PU podem ser variadas em uma grande faixa. As resinas podem ser obtidas com densidade

Autor para correspondência: Rita C. L. Dutra, AQI-CTA/IAE, Av. Prof. Mal. Eduardo Gomes 50, CEP: 12228-904, São José dos Campos, SP, Brasil. E-mail: ritad@iae.cta.br 
que variam de 6 a $1220 \mathrm{~kg} / \mathrm{m}^{3}$, podendo se apresentar como um elastômero de alta flexibilidade ou de maior dureza, ou como um plástico de engenharia ${ }^{[4]}$.

Como regra, um excesso de isocianato leva à obtenção de filmes mais duros, enquanto um excesso de poliol, outra matéria-prima da composição, resulta em adesivos de maior elasticidade. Deste modo, a caracterização dos adesivos quanto à sua estrutura química e teor de grupos funcionais, constitui um parâmetro importante para a avaliação prévia das propriedades desses materiais ${ }^{[3]}$.

Em geral, durante a manufatura de adesivos, a medida do índice de isocianato é um teste critico "in-process" para a completa reação de polimerização. Tradicionalmente, este teste pode ser realizado por titulação. Geralmente, as amostras são coletadas durante um determinado tempo de processo e encaminhadas ao laboratório de controle de qualidade para titulação. O resultado é calculado e comunicado ao operador de processo, que verificará a necessidade de ajuste.

Existem muitas desvantagens neste tipo de processo. $\mathrm{O}$ primeiro é o tempo gasto para a emissão de resultados que, excede o requerido para o processo após amostragem. Se a reação não seguir como o esperado, o produto pode ser perdido antes de um ajuste ser realizado. Este tempo é a maior desvantagem. Se a reação se completar, o processo não pode ser finalizado, a não ser que se admita o risco, antes de obter o dado analítico. Outra desvantagem é o fato de que a analise por titulação é visual, sujeita a erros de interpretação de analistas, e limitada em informação Esta análise não mostra problemas com o produto se a questão não afetar a medida do NCO. Finalmente, a compra e descarte de reagentes químicos utilizados na titulação são processos que envolvem custos consideráveis .

A espectroscopia no infravermelho (IR) tende a tornarse um método alternativo para substituir a determinação de $\mathrm{NCO}$ em adesivos, pois oferece vantagens como rapidez, simplicidade de operação de equipamento, não havendo a necessidade de utilização de reagentes químicos nocivos.

A espectroscopia IR pode ser utilizada para a identificação inequívoca da maioria dos adesivos ${ }^{[5,6]}$. No entanto, a quantificação destes componentes requer a elaboração de uma curva analítica que relacione a intensidade das bandas características ao teor de seus grupos funcionais correspondentes, determinado por outro método. Em geral, os métodos MIR estão em maioria em relação aos desenvolvidos na região NIR ${ }^{[7]}$.

Segundo a literatura ${ }^{[7,8]}$, a determinação por IR de NCO em poliuretanos consiste na medida da banda analítica em $2270 \mathrm{~cm}^{-1}$. O uso de banda referência têm sido citado ${ }^{[8]}$. Segundo Urbanski ${ }^{\mathrm{i}]}$, a banda em $2950 \mathrm{~cm}^{-1}$ do grupo $\mathrm{CH}_{3}$, pode ser usada como referência para essa determinação, entretanto, um cuidado deve ser tomado pois o erro citado é de $10 \%$, provavelmente por essa banda de referência ser muito intensa.

N.Sheikh e colaboradores ${ }^{[8]}$ usaram a espectroscopia FT-IR para monitorar a reação de cura de um pré-polímero uretânico com a umidade do ar. Foi utilizada a banda analítica em $2270 \mathrm{~cm}^{-1}$ (referente ao NCO) e $2921 \mathrm{~cm}^{-1}$ (referente a $\mathrm{CH}_{2}$ ) como banda de referência. Foi concluído que somente a utilização da umidade não era suficiente para cura com endurecimento do material e a utilização do catalisador 1,8-diazobicilo(5.4.0) undec-7-eno (DBU) mostrou-se efetiva na cura do adesivo ${ }^{[8]}$.

Metodologias MIR/NIR têm sido aplicadas a diferentes materiais, constituindo uma importante linha de pesquisa desenvolvida em nosso Centro de Pesquisa ${ }^{[9,10]}$. Entretanto, não foram ainda utilizadas para a quantificação de $\mathrm{NCO}$ em adesivos.

Portanto, considerando, que para a análise NIR existe um menor número de publicações, e nossos laboratórios (IAE/ Rohm and Haas) possuem espectrômetro IR, em ampla faixa espectral, e acessórios de última geração, nesse trabalho foi desenvolvido um método para a determinação de $\mathrm{NCO}$ em adesivos PU, utilizados em aplicações de laminados impressos e não impressos como PETP, PA, PP, PE (incluindo tipos EVA), utilizando-se banda relativa na região NIR, para correção de espessura e técnica ATR/diamante, com volume fixo, acessório não comum aos laboratórios, como fator diferencial às metodologias convencionais na região MIR.

\section{Experimental}

Adesivos fabricados na Rohm and Haas Química Ltda, com teores conhecidos de NCO, determinados por método analítico por via úmida, de acordo com a norma ${ }^{[1]}$ ASTM D2572 para amostras finais e amostras retiradas durante o processo de fabricação, foram utilizados nesse trabalho como amostras de referência e são mostrados na Tabela 1.

\section{Análise por FT-IR}

\section{MIR}

As amostras foram analisadas em espectrômetro FT-IR Avatar da ThermoNicolet (resolução $4 \mathrm{~cm}^{-1}$, ganho 4, 4000 a $400 \mathrm{~cm}^{-1}, 32$ varreduras), em volume fixo com ATR de diamante, nos laboratórios da Rohm and Haas Química Ltda.

A Figura 1 mostra o acessório ATR contendo ZnSe e diamante, utilizado neste trabalho. O alto índice de refração do elemento ATR é adequado para obtenção de espectros de excelente qualidade para a maior parte das substâncias, com grau de penetração da radiação na amostra em torno de 1-2 $\mu \mathrm{m}$. A dureza e a estabilidade química do diamante permite a obtenção direta de espectros de material em fase líquida, com bom contato entre a amostra e o cristal.

\section{NIR}

As amostras foram analisadas em espectrômetro FT-IR Spectrum 2000 PerkinElmer (resolução $4 \mathrm{~cm}^{-1}$, ganho 1, 7000 a $4000 \mathrm{~cm}^{-1}, 40$ varreduras), sob a forma de filme líquido. 
Tabela 1. Características das amostras AD-PU estudadas.

\begin{tabular}{|c|c|c|c|}
\hline Amostras & Tipo de amostras & $\begin{array}{c}\text { Teor de NCO } \\
(\% \mathrm{~m} / \mathbf{m}) \\
\text { (mínimo/máximo) }\end{array}$ & Aplicação \\
\hline Mor-Free ${ }^{\mathrm{TM}} 403 \mathrm{~A}$ & Meio fabricado e final & $17,50 / 18,30$ & $\begin{array}{l}\text { É um sistema de adesivo poliuretano de bi-componentes, sem solventes, } \\
\text { aplicados a baixa temperatura. O Mor-Free }{ }^{\mathrm{TM}} 403 \mathrm{~A} \text { é adequado para la- } \\
\text { minados de nylon, alumínio poliéster, polietileno e outros substratos de } \\
\text { embalagens não impressos ou impressos no reverso }\end{array}$ \\
\hline Adcote $^{\mathrm{TM}} 775 \mathrm{~A}$ & Meio fabricado e final & $2,40 / 2,70$ & $\begin{array}{l}\text { É um sistema de adesivo poliuretano de dois componentes, tendo como } \\
\text { solvente Acetato de Etila, com média quantidade de sólidos. O Adcote } \\
\text { 775A é adequado para laminados de PETP, PA, PP, PE (incluindo tipos } \\
\text { EVA) como bem metalizados e estruturas de folhas laminadas, filmes } \\
\text { que podem ser impressos com convenientes links para laminação }{ }^{[13]} \text {. }\end{array}$ \\
\hline Adcote $^{\mathrm{TM}} 534 \mathrm{~A}$ & Meio fabricado e final & $1,54 / 1,94$ & $\begin{array}{l}\text { O Adcote }{ }^{\mathrm{TM}} 534 \mathrm{~A} \text { é o componente poliuretano poliéster, terminado em } \\
\text { isocianato, de um sistema adesivo bi-componente de altos sólidos. O uso } \\
\text { do Catalisador 534A é necessário para se obter à cura total do adesivo. } \\
\text { Este adesivo adere a uma grande variedade de substratos, com exce- } \\
\text { lente resistência química e térmica. O Adcote }{ }^{\mathrm{TM}} 534 \mathrm{~A} \text { é especialmente } \\
\text { indicado para embalagens assépticas, onde a estrutura laminada pode } \\
\text { ser submetida a exposições prolongadas do peróxido de hidrogênio. O } \\
\text { Adcote }{ }^{\mathrm{TM}} 534 \mathrm{~A} \text {, com o Catalisador } 532 \mathrm{~B} \text {, pode ser usado em estruturas } \\
\text { que requerem alta resistência térmica }^{[14]} \text {. }\end{array}$ \\
\hline Adcote $^{\mathrm{TM}} 675 \mathrm{~A}$ & Meio fabricado e final & $2,00 / 2,25$ & $\begin{array}{l}\text { É um sistema de adesivo poliuretano de dois componentes, com base de } \\
\text { solvente com alta resistência química e ao calor e, que pode ser aplicado } \\
\text { com alto teor de sólidos. Adcote }{ }^{\mathrm{TM}} 675 \text { A é adequado para a laminação } \\
\text { de filmes tais como PEPT, PP, PE (incluindo tipos de EVA), PA também } \\
\text { na forma de estruturas metalizadas e folha de alumínio. Os filmes devem } \\
\text { ser impressos com tintas adequadas para laminação. Este adesivo tem } \\
\text { alta resistência ao calor e a produtos, para aplicações em embalagens } \\
\text { tais como embalagens onde o produto é cozido dentro da embalagem } \\
\text { (boil-in-bag), para uso com materiais agressivos tais como concentrados } \\
\text { de tomate, sabões e cosméticos }\end{array}$ \\
\hline
\end{tabular}

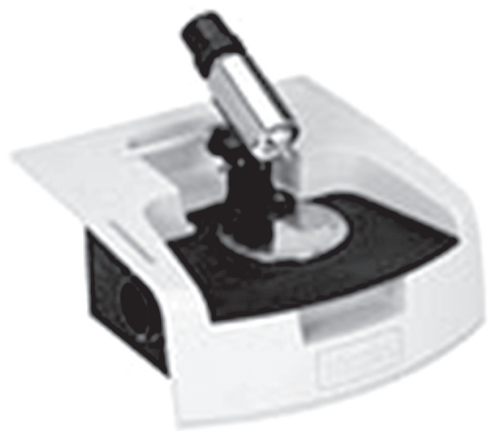

Figura 1. Acessório ATR utilizado neste trabalho.

\section{Resultados e Discussão}

\section{Análise por FT-MIR}

A Figura 2 inclui os espectros FT-IR, na região MIR de alguns adesivos estudados. A banda analítica escolhida, $2270 \mathrm{~cm}^{-1}$ é atribuída ao grupo NCO Os pontos de linha de base usados foram: $2457 \mathrm{e} 2106 \mathrm{~cm}^{-1}$.

Pode ser observado que há um aumento na intensidade da banda analítica com o aumento no teor de NCO nas amostras de adesivo. Essas características indicam que o método é adequado para quantificar o teor de NCO nas amostras de adesivos estudadas ${ }^{[12-15]}$
Para análise quantitativa MIR, os valores de absorvância em $2270 \mathrm{~cm}^{-1}$, foram relacionados ao teor respectivo de $\mathrm{NCO}$, sem a necessidade do uso de banda de referência para correção de espessura, em função da banda estar localizada em região espectral sem interferência de outras absorções.

A Tabela 2 inclui os valores de $\mathrm{A}_{2270}$ em função do teor de NCO (\%). Os valores de absorvância representam a mediana $(\mu)$ de 5 valores $^{[16]}$.

O desvio médio padrão, $\sigma_{\mu}$, da absorvância mediana foi calculado segundo a Equação $1^{[17]}$ :

$$
\sigma_{\mu}=\sigma / \sqrt{ } \mathrm{n}
$$

onde $\sigma$ é o desvio padrão e n, o número de medidas.

$\sigma$ é dado por:

$\sigma=\mathrm{K}_{\mathrm{R}} \cdot \mathrm{R}$

onde $\mathrm{K}_{\mathrm{R}}$ é o coeficiente para cálculo do desvio padrão de uma faixa de valores (para 5 experimentos $K_{R}=5$ ) e $R$, a diferença entre o maior e menor valor;

$$
\mathrm{R}=\mathrm{Xn}-\mathrm{X}_{1}
$$

O erro relativo para cada amostra analisada foi determinado por meio da Equação 4:

$$
\text { Erro relativo }(\%)=\left(\sigma_{\mu} / \mu\right) \times 100
$$


A Figura 3 mostra a curva analítica (calibração) $A_{2270}$ versus \% de NCO dos AD-PU: uma boa relação linear é obtida $(\mathrm{R}=0,995)$. Da curva de calibração, dada pela análise MIR, a seguinte relação é proposta:

$$
y=0,048+0,042 x
$$

onde y é o valor mediano da absorvância $\mathrm{A}_{2270} \mathrm{e}$ x é o valor de $\% \mathrm{NCO}$ nas amostras AD-PU.

$\mathrm{O}$ valor mediano do erro relativo correspondente à metodologia MIR é igual a $0,63 . \%$, que está dentro dos limites de precisão da metodologia IR ( $\leq 2 \%)$, de acordo com Hórak ${ }^{[16]}$.

\section{Análise por FT-NIR}

A Figura 4 inclui os espectros FT-IR, na região NIR de alguns adesivos estudados. As bandas escolhidas, analítica e de referência, 4063 e $4348 \mathrm{~cm}^{-1}$ estão próximas à região de bandas de combinação de grupos $\mathrm{C}$-H e primeiro sobretom do grupo $\mathrm{NCO}^{[18]}$. Os pontos de linha de base usados foram 4532 e $3909 \mathrm{~cm}^{-1}$

Valores de absorvância relativa $\mathrm{A}_{4063} / \mathrm{A}_{4348}$, mediana $(\mu)$ de cinco (5) análises, foram calculados e colocados em função do teor de NCO para contornar a variação de espessura ${ }^{[16]}$. Os

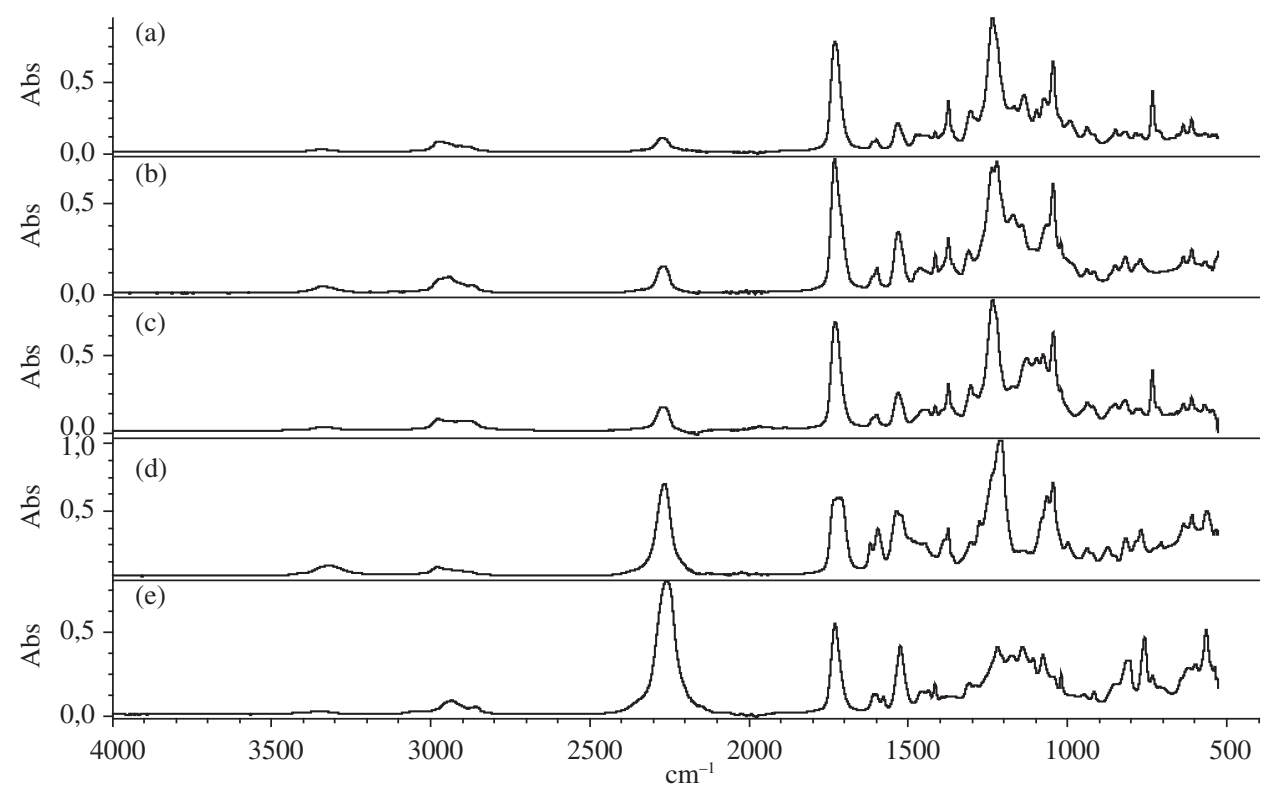

Figura 2. Espectros FT-MIR de AD-PU de diferentes teores $(\% \mathrm{~m} / \mathrm{m})$ de NCO - a) Adcote $^{\mathrm{TM}} 534 \mathrm{~A}$ - NCO 1,66; b) Adcote $^{\mathrm{TM}} 675 \mathrm{~A}$ - NCO 2,42; c) Adcote $775 \mathrm{~A}^{\mathrm{TM}}$ - NCO 2,51; d) Mor-Free ${ }^{\mathrm{TM}} 403 \mathrm{~A}$ - NCO 13,38; e e) Mor-Free $^{\mathrm{TM}} 403 \mathrm{~A}$ - NCO 17,52.

Tabela 2. Dados FT-MIR de amostras AD-PU com diferentes teores de NCO

\begin{tabular}{cccc}
\hline NCO $(\boldsymbol{\%} \mathbf{~ m} / \mathbf{m})$ & Valor mediano $(\mathbf{M I R}) \mathbf{A}_{\mathbf{2 2 7 0}} \mathbf{c m}^{\mathbf{1}}$ & Desvio médio padrão & Desvio relativo (\%) \\
\hline 1,57 & 0,092 & 0,001 & 1,05 \\
1,60 & 0,095 & 0,001 & 0,81 \\
1,64 & 0,085 & 0,001 & 0,68 \\
1,66 & 0,094 & 0,001 & 0,41 \\
2,23 & 0,144 & 0,001 & 0,40 \\
2,27 & 0,145 & 0,001 & 0,52 \\
2,27 & 0,149 & 0,001 & 0,66 \\
2,32 & 0,143 & 0,001 & 0,27 \\
2,51 & 0,151 & 0,001 & 0,64 \\
2,73 & 0,156 & 0,001 & 0,25 \\
3,77 & 0,218 & 0,001 & 0,62 \\
8,42 & 0,479 & 0,003 & 0,68 \\
8,65 & 0,472 & 0,001 & 0,29 \\
16,20 & 0,730 & 0,007 & 1,00 \\
16,30 & 0,692 & 0,006 & 0,86 \\
17,52 & 0,797 & 0,002 & 0,22 \\
17,71 & 0,776 & 0,006 & 0,79 \\
17,74 & 0,753 & 0,004 & 0,49 \\
\end{tabular}


valores de desvio médio padrão e relativo foram calculados de acordo com as equações já citadas (Tabela 3).

A Figura 5 mostra a curva analítica (calibração) $\mathrm{A}_{4063} / \mathrm{A}_{4348}$ vs \% de NCO dos AD-PU: uma boa relação linear é obtida $(\mathrm{R}=0,961)$. Da curva de calibração, dada pela análise NIR, a seguinte relação é proposta:

$$
y=0,646+0,034 x
$$

onde y é o valor mediano da absorvância $\mathrm{A}_{4063} / \mathrm{A}_{4348}$ e x é o valor de \% NCO nas amostras AD-PU.

$\mathrm{O}$ valor mediano do erro relativo correspondente à metodologia NIR é igual a $0,54 \%$, que está dentro dos limites de precisão da metodologia IR $(\leq 2 \%)$, de acordo com Hórak ${ }^{[12]}$.

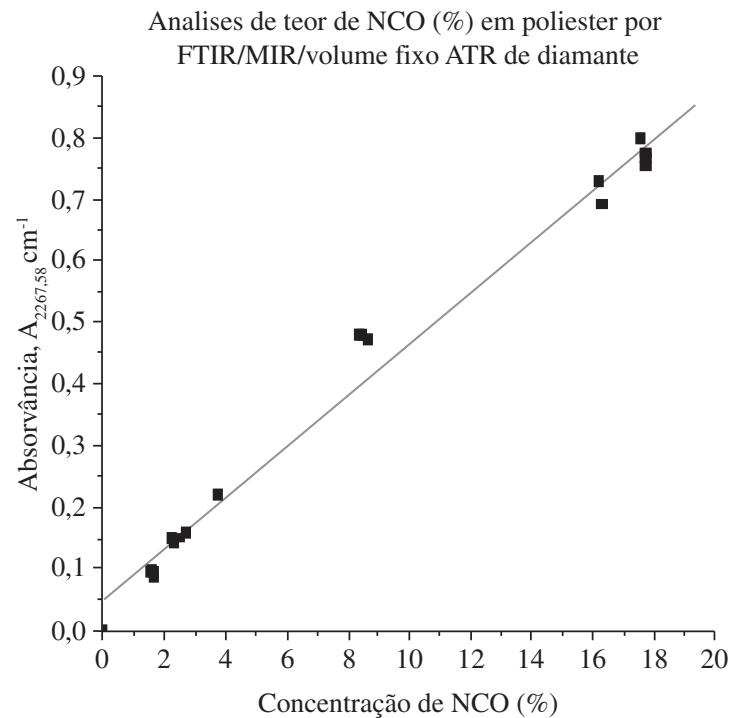

Figura 3. Valores medianos FT-MIR da absorvância $\mathrm{A}_{2270} \mathrm{~cm}^{-1} v s$ valores de $\mathrm{NCO}(\% \mathrm{~m} / \mathrm{m})$ das amostras AD-PU.
As amostras AM001 à AM007 foram utilizadas para testar as curvas obtidas com o uso das metodologias MIR e NIR. A precisão dos dados obtidos das análises por ambos procedimentos (MIR e NIR) são mostrados na Tabela 4.

\section{Conclusão}

Um novo método FT-MIR/NIR foi desenvolvido e comparado ao método titulométrico para determinação do teor de NCO nas amostras de adesivos poliuretânicos (AD-PU).

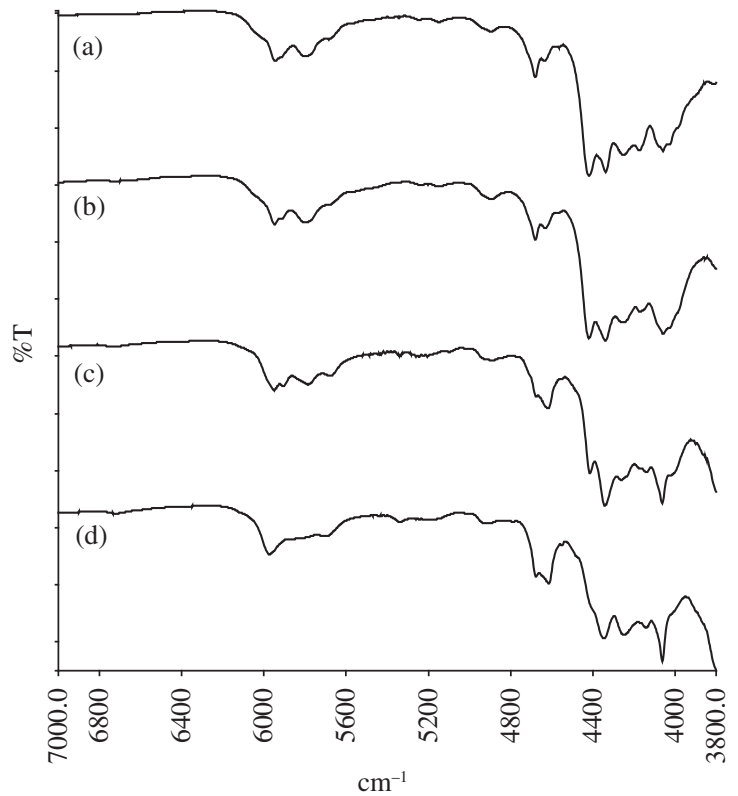

Figura 4. Espectros FT-NIR de AD-PU de diferentes teores $(\% \mathrm{~m} / \mathrm{m})$ de $\mathrm{NCO}$ - a) Adcote ${ }^{\mathrm{TM}} 534 \mathrm{~A}$ - NCO 1,55; b) Adcote $775 \mathrm{~A}^{\mathrm{TM}}$ - NCO 2,56; c) Mor-Free ${ }^{\mathrm{TM}} 403 \mathrm{~A}$ - NCO 12,0; e d) Mor-Free ${ }^{\mathrm{TM}} 403 \mathrm{~A}-\mathrm{NCO} 17,80$.

Tabela 3. Dados FT-NIR de amostras AD-PU com diferentes teores de NCO.

\begin{tabular}{cccc}
\hline NCO $(\boldsymbol{\%} \mathbf{~ m} / \mathbf{m})$ & Valor mediano $(\mathbf{N I R}) \mathbf{A}_{\mathbf{4 0 6 3}} / \mathbf{R}_{\mathbf{4 3 4 8}}$ & Desvio médio padrão & Desvio relativo (\%) \\
\hline 1,57 & 0,649 & 0,005 & 0,74 \\
1,60 & 0,653 & 0,003 & 0,53 \\
1,64 & 0,646 & 0,003 & 0,54 \\
1,66 & 0,658 & 0,007 & 1,02 \\
2,23 & 0,642 & 0,003 & 0,48 \\
2,27 & 0,641 & 0,003 & 0,42 \\
2,27 & 0,680 & 0,003 & 0,40 \\
2,51 & 0,866 & 0,012 & 1,40 \\
2,73 & 0,834 & 0,011 & 1,29 \\
3,77 & 0,892 & 0,017 & 1,94 \\
8,42 & 1,046 & 0,015 & 1,45 \\
8,65 & 0,985 & 0,022 & 2,21 \\
16,20 & 1,143 & 0,004 & 0,37 \\
16,30 & 1,228 & 0,011 & 0,89 \\
17,52 & 1,257 & 0,34 \\
17,74 & 1,252 & 0,004 & 0,25 \\
17,74 & 1,235 & 0,005 & 0,39 \\
\end{tabular}


Tabela 4. Dados de precisão de análise de FT-NIR e FT-MIR.

\begin{tabular}{|c|c|c|c|c|c|c|c|c|}
\hline \multirow[t]{2}{*}{ Amostra } & \multirow{2}{*}{$\begin{array}{c}\text { Código } \\
\text { das } \\
\text { amostras }\end{array}$} & \multicolumn{2}{|c|}{ Absorvância } & \multicolumn{3}{|c|}{$\begin{array}{c}\text { Concentração de NCO por método } \\
(\% \mathrm{~m} / \mathrm{m})\end{array}$} & \multicolumn{2}{|c|}{$\begin{array}{c}\text { Desvio } \\
\text { relativo }(\%)\end{array}$} \\
\hline & & $\operatorname{MIR}\left(\mathrm{A}_{2270 \mathrm{~cm}^{-1}}\right)$ & relativa NIR $\left(\mathrm{A}_{4063} / \mathrm{A}_{4348}\right)$ & titulométrico & MIR & NIR & MIR & NIR \\
\hline Adcote $^{\mathrm{TM}}$ 534A & AM001 & 0,111 & 0,696 & 1,56 & 1,50 & 1,47 & 1,06 & 0,96 \\
\hline Adcote $^{\mathrm{TM}} 534 \mathrm{~A}$ & AM002 & 0,115 & 0,699 & 1,69 & 1,60 & 1,56 & 0,92 & 1,02 \\
\hline Adcote $^{\mathrm{TM}} 534 \mathrm{~A}$ & AM003 & 0,121 & 0,703 & 1,78 & 1,74 & 1,68 & 0,94 & 0,94 \\
\hline Adcote $^{\mathrm{TM}} 675 \mathrm{~A}$ & AM004 & 0,133 & 0,711 & 2,04 & 2,02 & 1,91 & 0,72 & 0,33 \\
\hline Adcote $^{\mathrm{TM}} 775 \mathrm{~A}$ & AM005 & 0,795 & 1,248 & 17,77 & 17,79 & 17,71 & 0,37 & 0,61 \\
\hline Mor-Free $^{\mathrm{TM}} 403 \mathrm{~A}$ & AM006 & 0,783 & 1,239 & 17,53 & 17,50 & 17,44 & 0,58 & 1,12 \\
\hline Mor-Free $^{\mathrm{TM}} 403 \mathrm{~A}$ & AM007 & 0,813 & 1,279 & 18,30 & 18,21 & 18,62 & 0,11 & 0,50 \\
\hline
\end{tabular}

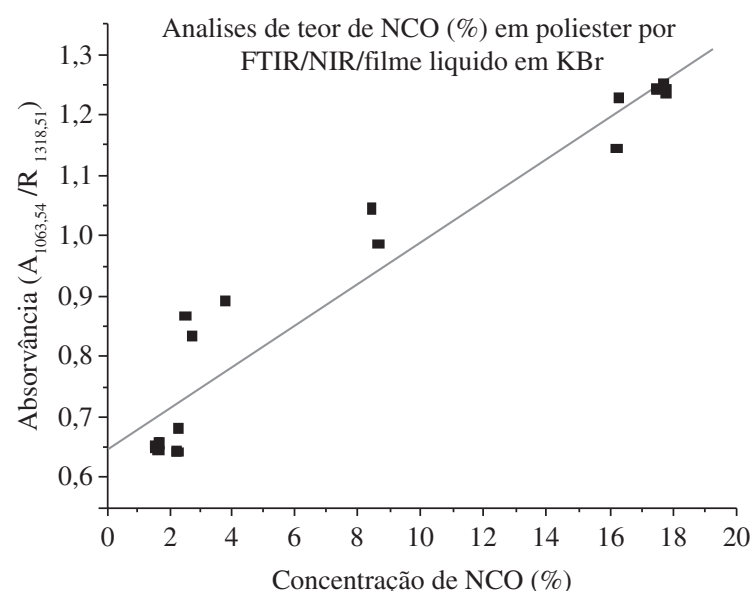

Figura 5. Valores medianos FT-NIR da absorvância relativa $A_{4063} / A_{4348,} \mathrm{~cm}^{-1}$ vs valores de $\mathrm{NCO}(\% \mathrm{~m} / \mathrm{m})$ das amostras AD-PU.

Segundo a literatura, a determinação MIR de $\mathrm{NCO}$ em poliuretanos consiste na medida da banda analítica em $2270 \mathrm{~cm}^{-1}$. O uso de banda referência têm sido citado, entretanto, um cuidado deve ser tomado pois o erro da metodologia proposta encontrada na literatura é de $10 \%$, provavelmente em função das bandas de referência (grupos $\mathrm{CH}_{3}$ e $\mathrm{CH}_{2}$ ) serem muito intensas.

Nesse trabalho, além do uso de técnica ATR/diamante, com volume fixo, acessório não comum aos laboratórios, como fator diferencial às metodologias convencionais na região MIR, a banda em $2270 \mathrm{~cm}^{-1}$ foi usada, por ser a analítica, mas não associada à absorções intensas de referência para correção de espessura. Em função da banda em $2270 \mathrm{~cm}^{-1}$ estar localizada em região espectral sem interferência de outras absorções, o erro foi bem menor, $0,63 \%$, em comparação ao citado na literatura, $10 \%$.

Desde que o principal objetivo deste trabalho foi desenvolver um método rápido e preciso para controle de qualidade, é possível considerar que os métodos MIR e NIR são equivalentes, podendo ser usados onde maior precisão é requerida, desde que o erro relativo não excede $2 \%$ da escala de transmitância, de acordo com os limites de precisão do espectrômetro usado.

Foi observado que ambos os métodos, MIR e NIR apresentam essas e outras vantagens, como o fator limpeza (sem necessidade de limpeza de vidraria) em relação ao método titulométrico. Os dados obtidos pelos métodos titulométrico, MIR e NIR mostraram boa concordância.

\section{Referências Bibliográficas}

1. Edwards, B. H. - "Structural Adhesives - Chemistry and Technology", Polyurethane Structural Adhesives, cap. 4, Plenum Press, New York (1986).

2. Sarantópolus, C. I. G. L.; Oliveira, L. M.; Padula, M.; Coltro, L. Alves, R. M. V. \& Garcia, E. E. C. - "Embalagens Plásticas Flexíveis - Principais Polímeros e Avaliação de Propriedades", cap. I, CETA/ITAL (2002).

3. Vilar, Walter Dias - "Química e Tecnologia dos Poliuretanos", Vilar Poliuretanos, $3^{\mathrm{a}}$ ed (2005).

4. Woods, G. - "The ICI Polyurethanes Book", John Wiley, New York (1990).

5. Malucelli, G.; Priola, A.; Ferrero, F.; Quaglia, A.; Frigione M. \& Carfagna, C. - International Journal of Adhesion \& Adhesives 25, p.87-91 (2005).

6. Henry, C. Y.; Jie Daí, K. \& Tan, E. - Journal of Applied Polymer Science, 102, 4284-4290 (2006)

7. Urbanski et al - "Handbook of analysis of synthetic polymers and plastics" John Wiley \& Sons, New York, (1977)

8. Sheikh, N.; Katbab, A. A. \& Mirzadeh, H - International Journal of Adhesion \& Adhesives 20, p.299-304 (2000).

9. Mattos, E. C; Moreira, E. D.; Dutra, R. C. L.; Diniz, M. F.; Ribeiro, A. P. \& Iha, K. - Quim. Nova, 27, p.540-544 (2004).

10. Dutra, R. C. L.; Oliveira, J. I. S.; Diniz, M. F.; Kawamoto, A. M. \& Keicher, T. - Propellants, Explosives, Pyrotechnics, 31, p.395400 (2006).

11. American Society for Testing and Materials - ASTM D2572-97 - Standard Test Method for Isocyanate Groups in Urethane Materials or Prepolymers.

12. Rohm and Haas Company, TDS - Mor-Free 403A/C-132-1 (Port).

13. Rohm and Haas Company, TDS - Adcote $775 \mathrm{~A}+775 \mathrm{C}$, II/2004 (2004).

14. Rohm and Haas Company, TDS - Adcote534A/534B532B (Port).

15. Rohm and Haas Company, TDS - Adcote 675 A/C (Port), 07/03 (2003).

16. A.L. Smith - "Applied Infrared Spectroscopy", John Wiley \& Sons, New York (1979).

17. M. Hórak; \& A. Vítek - "Interpretation and processing of Vibrational Spectra", John Wiley \& Sons, New York (1978).

18. Goddu, R. - Near-Infrared Spectrophotometry, Adv. Anal. Chem. Instrum., New York, v. 1, p. 347-425 (1960).

Enviado: 09/05/07

Reenviado: $30 / 08 / 07$

Aceito: $17 / 09 / 07$ 